\title{
Ergonomic Guidelines for Using Notebook Personal Computers
}

\author{
Susumu SAITO ${ }^{1 *}$, Bruno PICCOLI², Michael J. SMITH³ ${ }^{3}$ Midori SOTOYAMA ${ }^{1}$, \\ Glenn SWEITZER ${ }^{4}$, Maria Beatriz G. VILLANUEVA ${ }^{5}$ and Ryoji YOSHITAKE ${ }^{6}$
}

Technical Committee on Human-Computer Interaction, International Ergonomics Association (IEA)

${ }^{1}$ National Institute of Industrial Health, Nagao 6, Tama-ku, Kawasaki 214-8585, Japan

${ }^{2}$ Department of Occupational Health, Milan State University - ICP Hospital, Milan, Italy

${ }^{3}$ Department of Industrial Engineering, University of Wisconsin-Madison, Madison, WI 53706, USA

${ }^{4}$ Department of Building Services Engineering, Hong Kong Polytechnic University, Hung Hom, Kowloon, Hong Kong-SAR, China

${ }^{5}$ Occupational Safety and Health Center, Quezon City 1104, Philippines

${ }^{6}$ Human Factors, Yamato Lab., IBM Japan, 1623-14, Shimotsuruma, Yamato 242-8502, Japan

Received August 21, 2000 and accepted September 11, 2000

\begin{abstract}
In the 1980's, the visual display terminal (VDT) was introduced in workplaces of many countries. Soon thereafter, an upsurge in reported cases of related health problems, such as musculoskeletal disorders and eyestrain, was seen. Recently, the flat panel display or notebook personal computer (PC) became the most remarkable feature in modern workplaces with VDTs and even in homes. A proactive approach must be taken to avert foreseeable ergonomic and occupational health problems from the use of this new technology. Because of its distinct physical and optical characteristics, the ergonomic requirements for notebook PCs in terms of machine layout, workstation design, lighting conditions, among others, should be different from the CRT-based computers. The Japan Ergonomics Society (JES) technical committee came up with a set of guidelines for notebook PC use following exploratory discussions that dwelt on its ergonomic aspects. To keep in stride with this development, the Technical Committee on Human-Computer Interaction under the auspices of the International Ergonomics Association worked towards the international issuance of the guidelines. This paper unveils the result of this collaborative effort.
\end{abstract}

Key words: Ergonomics, Guidelines, VDT, Notebook PC, Laptop PC, Flat Panel Display (FPD)

\section{Introduction}

While advanced technologies can enrich our lifestyles, they can also contribute to problems such as fatigue and stress. Electronic displays used for word processing, internet access including video clips and other similar visual tasks are no exception. Numerous ergonomic guidelines have been proposed in order to promote comfortable use of displays. Many of these guidelines are for cathode-ray tube

*To whom correspondence should be addressed.
(CRT) displays, which are based on the same principle used for television. Recently, however, flat panel displays (FPDs) are being used widely in place of CRTs, in offices, at home, and even in mobile devices by people on the move. This type of display is typically called a notebook personal computer (PC). Notebook PCs with FPDs are extremely portable, and can be used anywhere, at any time. FPDs differ from CRTs in form, features, and optical characteristics, and therefore necessitate ergonomic guidelines that differ from those used in the past.

In October 1996, the Japan Ergonomics Society (JES) 


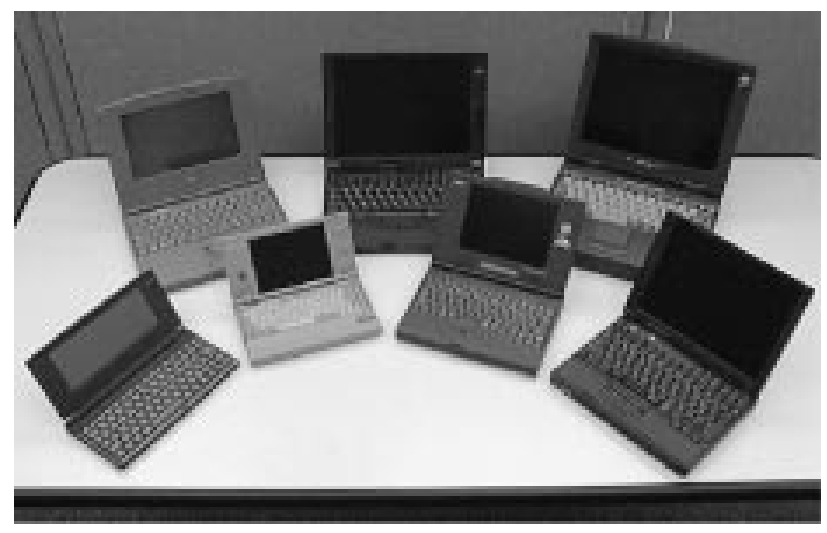

Fig. 1. Various types of notebook PCs.

established a "Committee for Flat Panel Display Ergonomics". The goal of the committee was to propose ergonomic guidelines for the use of products with FPDs, such as liquid crystal display (LCD). Many products use FPDs, but the committee started its activities by utilizing the accumulated ergonomic know-how and experience related to notebook PCs with back-light LCD. The members of the drafting committee in JES are listed in Appendix 2 of this paper, and the Japanese guidelines were published in 1998 (Japan Ergonomics Society (1998), Ergonomic guidelines for using notebook personal computers, (in Japanese), pp. 1-28, Institute for Science of Labour, Kawasaki).

The authors of this paper, members of the Technical Committee on Human-Computer Interaction, which worked under the auspices of the Science and Technology Committee of the International Ergonomics Association (IEA) have discussed on the international version of the guidelines based on the Japanese version. The results are published here in this issue of the journal, Industrial Health. All the members of the Technical Committee on Human-Computer Interaction of IEA are listed in Appendix 3.

The information provided below describes methods for effectively using devices with FPDs. The authors hope that these guidelines are valuable resources for existing and prospective users of notebook PC, as well as designers for those developing FPD devices with the goal of "HumanCentred Design" in mind.

\section{Working with Displays}

Computers with electronic displays began to be used widely in workplaces from the late ' 70 s to the early ' 80 s. Shortly after, several health problems, such as musculoskeletal disorders and eyestrain, emerged and in response considerable research in ergonomics and occupational health followed. The findings led to recommendations to prevent fatigue and health problems while increasing user comforts. These included addressing user needs through improved interfaces with display hardware and software as well as the workplace environment.

Display users often tend to view their work from a short viewing distance and with poor posture. That is, they stress their musculoskeletal systems. Moreover, the related increase in accommodation and convergence is intense and sometimes causes an overloading of these visual functions. Symptoms include eyestrain, asthenopia, and neck, shoulders and arms ache. In this regard it is important to note that when there is a conflict between vision and posture comfort, normally the subject (particularly if involved in occupational activities) privilege the first, being visual needs considered essential for a VDT performance. Consequently it is not so unusual that neck, shoulder and arms muscular disturbances are linked, at least partially, to visual problems. These symptoms may be exacerbated for users of, for example, large CRT displays on small desks, and notebook PCs with nondetachable keyboards. Each condition tends to constrain the posture to one fixed position. For such situations it is important to regulate the workload. Following are factors that need to be considered when working with displays:

- Workplace environmental factors, including thermal comfort, indoor air quality, and noise exposure

- Task and ambient illumination, including the display itself

- Posture, including desk, chair, keyboard, and workstation layout

- Average viewing distance (related both to characters and icons sizes)

- Office tasks and schedule

- Individual health

Holistic consideration of these factors can help improve the health and comfort of display users.

\section{Target Audience and Use of these Guidelines}

These guidelines were written mainly for the following types of readers:

- Those who are already using a notebook PC at work or at home (from beginners to experienced users)

- Those who are considering buying and then using a notebook PC

- Those who will be lent or given a notebook PC to use at work

- Those who are considering using a notebook PC instead of a desktop PC 
In addition to these types of readers, developers and retailers of notebook PCs, manufacturers of office equipment and furniture, and office designers will benefit from these guidelines.

Although we recommend that first-time readers read the entire document from beginning to end if practical, perusal of each chapter's title and the specific action steps (which only cover a few lines) alone are enough help. Starting out by glancing through the entire document can also help. Detailed explanations are included in various parts of the document in order to provide a deeper understanding of the issues. We also recommend that those in charge of purchasing equipment for workplaces, managers, and executives understand the detailed explanations as well.

\section{Guidelines for Notebook PC Users}

This chapter describes ergonomic guidelines for using notebook PCs. Notebook PCs are conveniently portable and can be used in a variety of locations, but this document assumes that a notebook PC is being used indoors, inside an office, home, or similar location. The outdoor use of notebook PCs requires a different set of guidelines. Since most of these guidelines apply to notebook model word processors as well, read " notebook PC" as " notebook word processor" if appropriate to your situation. These guidelines are divided into nine sections, each explaining concrete usage methods.

\subsection{Work Environment and Workstation Layout}

"Create an environment that fits your work"

a. Verify that your environment (lighting, temperature, noise, and so on) is appropriate for working. In particular, make sure that your notebook PC is set up in a location that is neither too bright nor too dark. For instance, make sure that you can adjust the level of lighting with blinds, curtains, or similar, particularly if you work with your back to a window.

b. Make sure to allocate enough space on your desk when placing a notebook PC.

\section{Explanation:}

Even when using a device that has been well-designed from an ergonomic point of view, if the working environment is poor, a person will not be able to work comfortably (of course this principle is not limited to notebook PCs). For instance, lighting cannot be too bright or too dark. The comfort zones for lighting, temperature, humidity, and noise level are given in Table 1, so try to set up your work

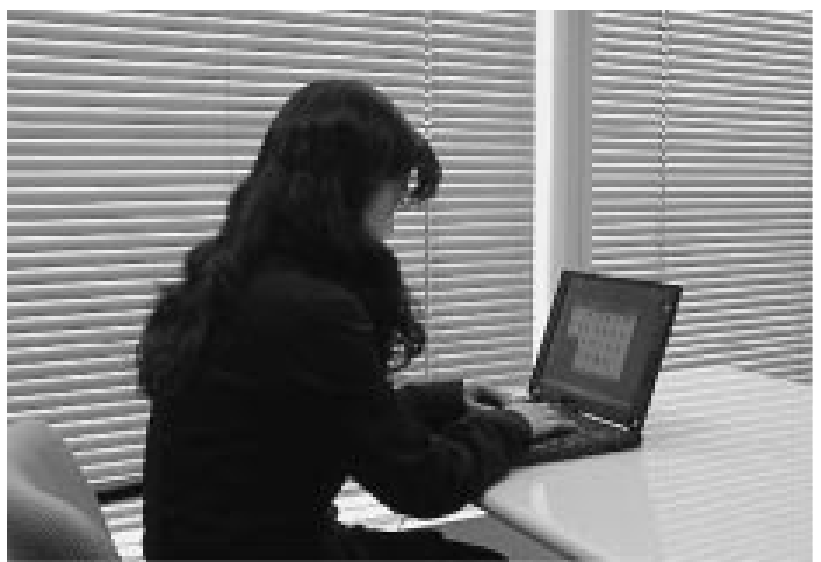

Fig. 2. When working near a window, balance the level of daylighting by adjusting blinds or other shading devices.

environment so that it falls within these ranges.

The ease of viewing a display is particularly affected by lighting conditions. Make sure that your display is oriented in such a way that no high-intensity objects (extremely bright objects) such as light sources and windows are reflected on the display. Since the lighting is generally darker in homes than in offices, when using a notebook PC at home at night, you may want to turn down the PC's brightness, contrast, or other settings (see 4.4). On the other hand, do not turn off all the surrounding lights and leave only the notebook PC's display on in order to help you concentrate on your work, or to make the display stand out more. Unless it makes the display extremely difficult to see, you should set the room lighting to a level that would be appropriate for reading a newspaper. In this regard the use of a localised light source (table lamp), equipped with a dimmer, is very recommended, particularly when paper documents have to be consulted. A good integration between general light and localised light, according to kind of activity and individual psycho-physical characteristics (personal orientations, age, ophthalmologic alterations, etc.), seem to play an important role in preventing illumination disturbances.

Notebook PCs are relatively small, so they do not take up much space when not in use. If someone is going to set up and work with the notebook PC, however, then it is important to make sure that ample space is provided on the desk. Set aside enough space that the notebook PC can be moved forward, backward, left, and right, in order to eliminate the necessity of holding the same posture for long periods of time.

Also, since pointing devices that come standard with notebook PCs emphasize portability, there are cases when a mouse is easier to use, depending on the tasks (see 4.5). 


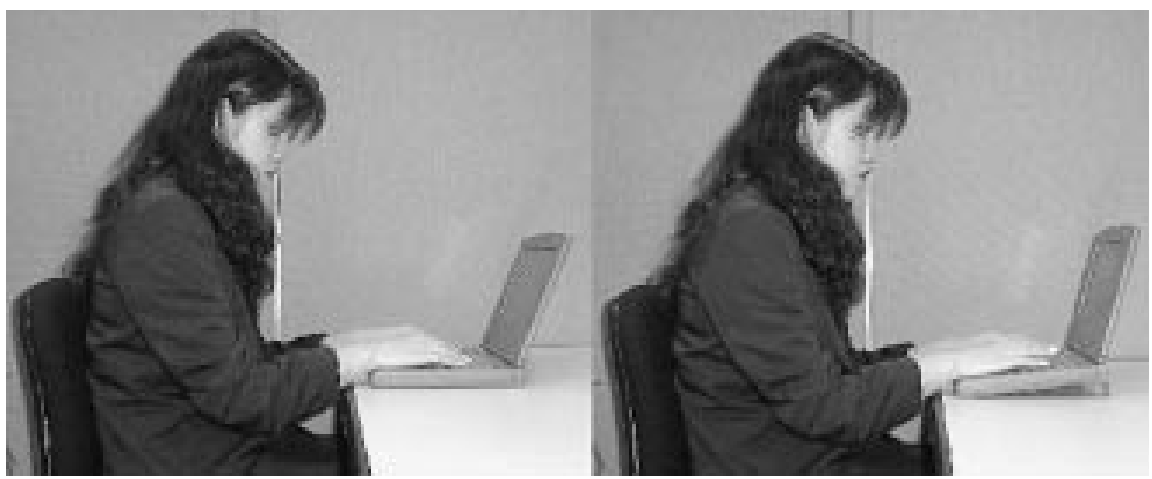

Fig. 5. The difference between keyboards without (left picture) and with (right picture) an angle-At this chair height and with this posture, the angled keyboard on the right is more natural because the wrists are straight.

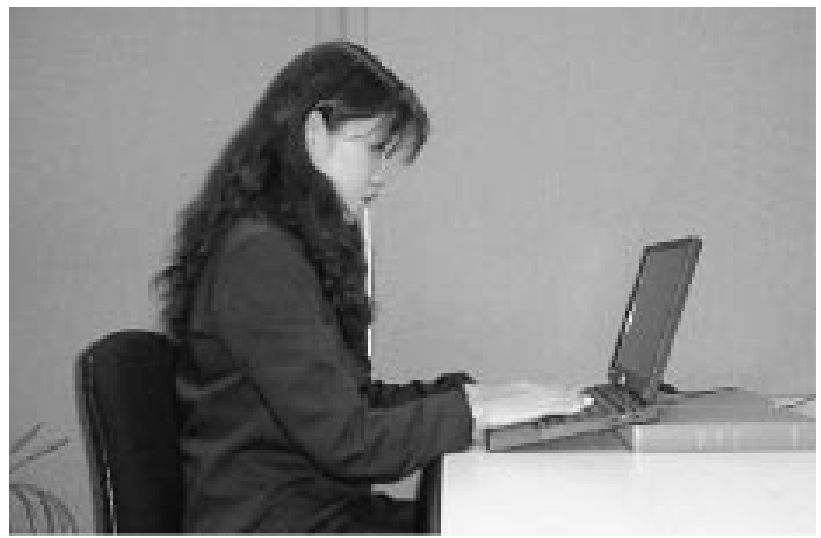

Fig. 6. Even if the notebook PC itself doesn't come with legs attached, you can add an angle by using a book.

elbow height.

\subsection{Keyboard}

"Set the keyboard to a desirable angle, and use a palm rest if necessary"

a. Adjust the angle of the keyboard based on your posture and preferences.

b. Make sure there is space in front of the keyboard for you to comfortably rest your wrists (this space can be on the desktop surface itself if the keyboard is thin).

c. If the keyboard seems difficult to use, use an external keyboard (see 4.9).

\section{Explanation:}

Desktop PC keyboards are usually angled so that when placed on a desk, the back is high and the front is low. There is also a type of keyboard whose key tops have shapes that are varied slightly from row to row, in order to provide a

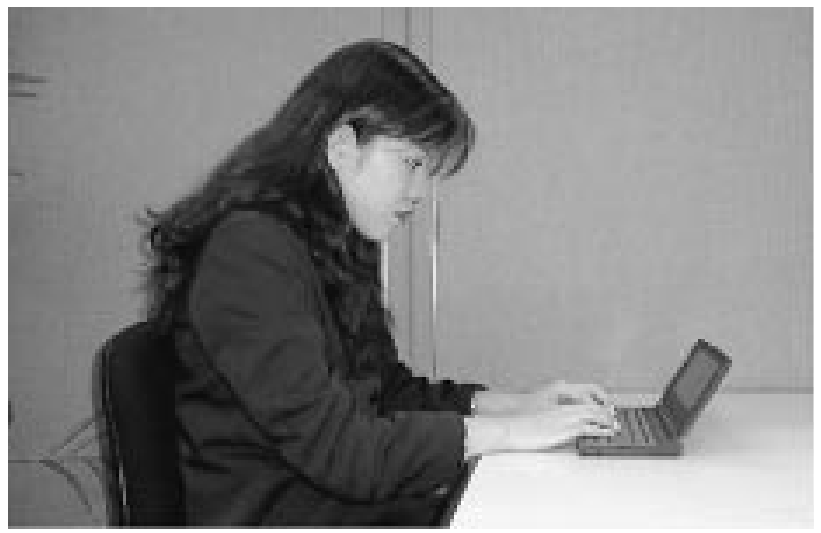

Fig. 7. Example of using the desktop surface as a palm rest.

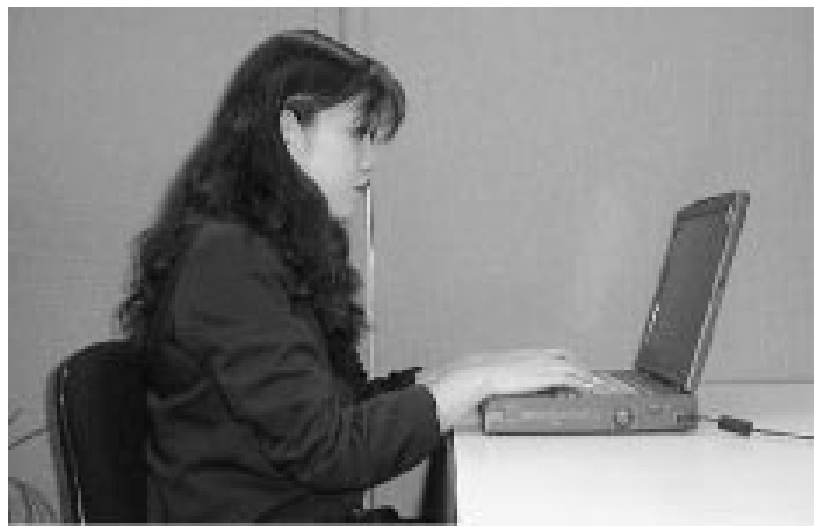

Fig. 8. Example of the palm rest getting in the way because the chair is too low.

snug fit with the fingers (this is called "step sculpturing"). In the case of notebook PC keyboards, however, the angle of the keyboard is designed to be parallel to the desktop 
Table 2. Necessary palm rest depth based on the length of the user's hands (Hand length: The length from the tip of the middle finger to the wrist for an open hand)

\begin{tabular}{lrrrrrr}
\hline Length of User's Hand (mm) & 160 & 170 & 180 & 190 & 200 & 210 \\
Minimum Necessary Depth (mm) & 39 & 45 & 52 & 59 & 65 & 72 \\
Recommended Depth (mm) & 60 & 67 & 73 & 80 & 87 & 93 \\
\hline
\end{tabular}

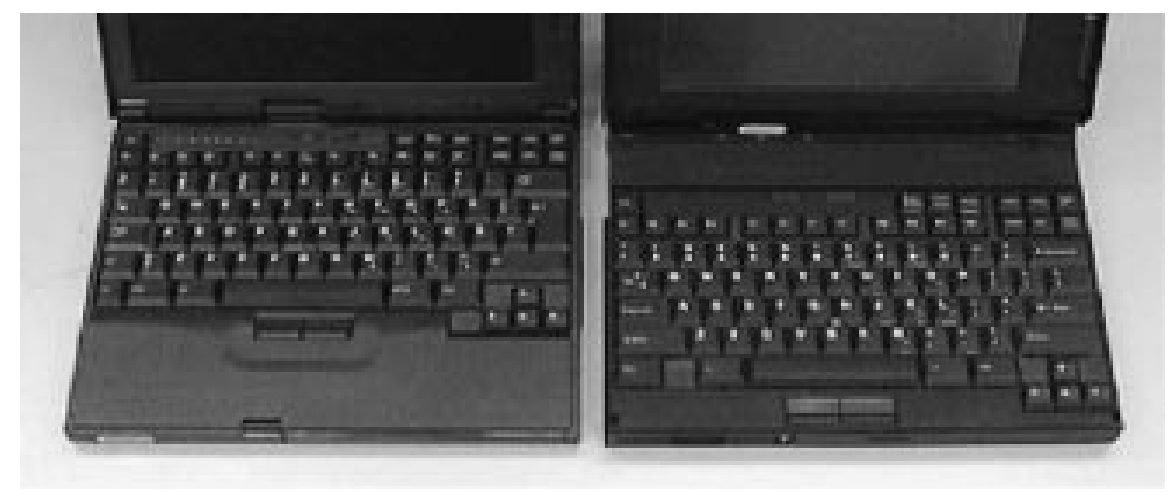

Fig. 9. Models with different depth palm rests.

surface, in order to keep the notebook PC as slim as possible. As a result, the shape of the key tops is almost flat in many cases. Some notebook PCs come with legs that allow you to add an angle, so check if your notebook PC has this sort of function first.

Even if a notebook PC does not come with this function built-in, you can improvise by placing an object such as a book or magazine underneath the notebook PC at the back. The best angle is one whereby the wrists are straight, and do not bend unnaturally. Although $5 \mathrm{deg}$. to $12 \mathrm{deg}$. is the generally suggested range of angles, the easiest angle to use differs depending on the individual's physique and working posture. Even for the same person, as posture changes throughout the course of prolonging working hours, the optimum angle will change as well, so there is no need to set a single standard angle for each person.

If your keyboard is not very thick, then you can use the desktop as a palm rest or arm rest. If the keys are very high off the desktop, however, you may want to use a book or towel to make your own palm rest. Commercial palm rests are also available. It is extremely important, however, that your palm rest does not get in the way when you type, so be careful when you set it up. Although many recent notebook PCs come with built-in palm rests, it should be noted that the necessary depth for a palm rest depends on the size of the user's hands. You can use the following table as a general guideline for determining the necessary palm rest depth based on your hand size:

\subsection{Display}

"Carefully adjust the display's tilt angle, brightness, contrast, and other settings in order to make it as easy to see as possible"

a. Adjust the display angle so that light sources such as ceiling lights, task lights, and windows are not reflected on the display.

b. If your notebook PC has knobs for setting display attributes such as brightness and contrast, adjust it to an easy-to-see brightness and hue setting that is not too dark.

c. A display's brightness and hue may vary depending on the viewing angle. Keep this in mind as you adjust the display angle for easy viewing. Avoid (or at least attenuate) the presence of bright light spots, even if very small, in the visual field. Display average luminance is $20-40 \mathrm{~cd} /$ $\mathrm{m}^{2}$ and "light spots" over $400 \mathrm{~cd} / \mathrm{m}^{2}$ could cause visual fatigue and decrease of effectiveness, while if over 1000 $\mathrm{cd} / \mathrm{m}^{2}$ intense ocular and visual disturbances could arise.

d. Optimum viewing conditions will change along with the information displayed on the display, the workplace environment, user fatigue and acclimatisation, and other factors. Repeat steps "a" to "c" as necessary.

e. If the display seems difficult to use, switch to an external monitor (refer to 4.9).

\section{Explanation:}

Display reflections vary by PC type. Reflections do not 


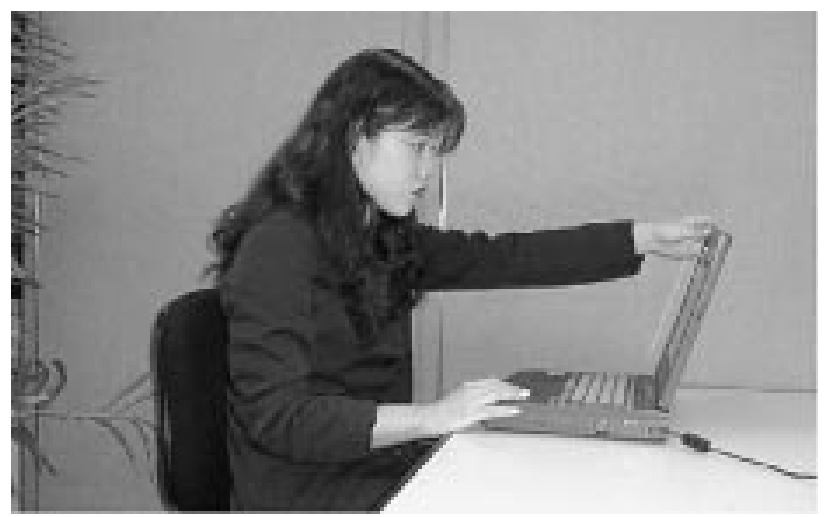

Fig. 10. Avoid reflections, and adjust the angle for ease of viewing.

stand out on higher performance models with low reflection rates. Reflections are particularly noticeable when nothing is displayed on a display, so check your notebook PC. If reflections stand out on the model you are using, then you will have to take particular care when adjusting its angle and location in order to avoid reflections of electric lights and other light sources.

The principal used in LCDs used in notebook PCs is different from that used for CRT displays. LCDs are superior to CRT displays in that the display is flat and has no curvature distortions, so there is no blur or smearing. On the other hand, with some models, brightness, hue, and other optical characteristics vary by viewing angle, so care must be taken. Since LCDs used for PCs are easiest to view from straight ahead, or from an angle slightly above straight-ahead, you should usually adjust the viewing angle of your display accordingly. The easiest viewing angle can vary depending on whether the display is showing a picture or photograph with an intermediate brightness level or many different colors, or whether it is only showing letters and symbols. Be sure to carefully adjust the viewing angle depending on what is displayed on the display.

Display brightness and contrast levels have the greatest effect on ease of viewing. Adjust the display to an optimum brightness level that is neither too bright nor too dark. The range of possible settings for notebook PCs is narrow, and some models cannot be set to the optimum brightness level. If this is the case, you may be able to increase visibility by turning a task light (a light such as a desktop stand lamp that is adjustable by hand, and that does not light up an entire room) on and off, or by changing the location. Since the optimum brightness setting varies with the brightness of lighting (illumination), if the display seems too dark (the contrast is too low), make the surroundings darker (turn down the lighting around the display). If the display seems too bright (the contrast is too high), make the surroundings brighter (turn up the lighting around the display). This will generally improve visibility.

In the case of a back-light LCD, the brightness of the back-light will stabilize approximately 20 minutes after it is turned on. Since the optimum brightness will vary depending on the information displayed, you should readjust it as necessary. For example, the display does not have to be very bright if you are reading and writing letters and symbols, but the brightness of the entire display should be increased for maximum visibility when displaying objects such as pictures and photographs. Also, keep in mind that the preferred brightness level varies by individual eyesight, age, experience, and other factors. Accordingly, make no sweeping comparisons with other people. Even the preferred brightness for a single individual may change after long periods of use, so be sure to readjust the brightness as necessary.

If the displayed text appears small and difficult to read, you can increase the font's point size, or use a software zoom function to select a zoomed display setting of $150 \%$ or so. Be sure to use your software's function settings to make sure the text is easy to read.

\subsection{Non-keyboard Input Devices}

"Use a mouse as your pointing device if at all possible"

a. If a mouse can be connected to your notebook PC, then do so as often as possible

b. Use a mouse pad whenever you use a mouse

c. When you cannot connect a mouse, make sure you understand the built-in pointing device, and use the pointing device appropriately

d. If you find yourself losing the pointer (such as the mouse cursor), then set up your notebook PC so that the pointer leaves a trail

\section{Explanation:}

The standard pointing devices that come connected to notebook PCs are designed to be usable anywhere outside the office. For this reason, when using a notebook PC in the office, you may find that the standard pointing device is not as easy to use as a mouse. Almost all notebook PCs can be connected to a mouse, so you should connect a mouse if at all possible when working in the office or at home. You can use both the standard pointing device and the mouse, and choose one or the other depending on the particular type of work you are doing at the time.

Standard notebook PC pointing devices come in a number 


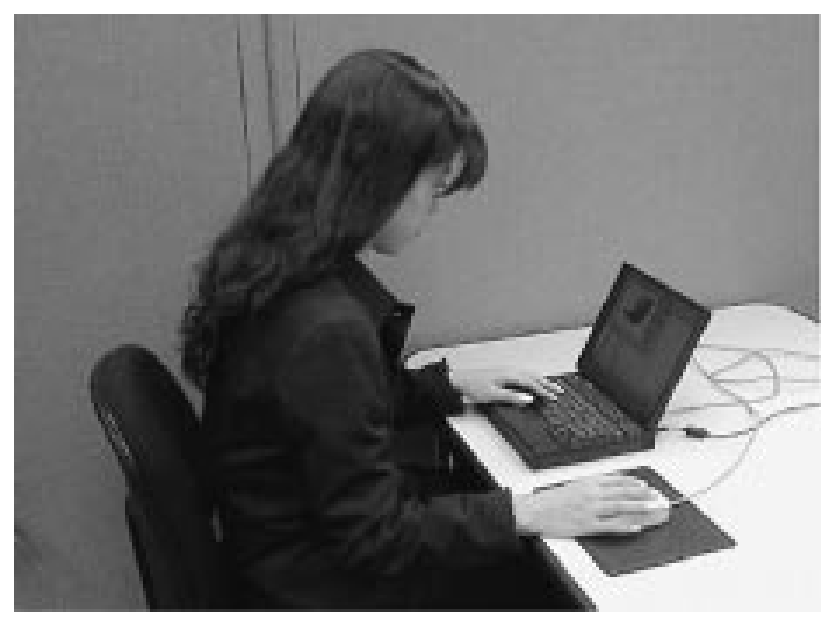

Fig. 11. Mouse connection example.

of different types, so you should check the type of device included with your notebook PC, and learn the correct use of that device in order to improve ease of use.

The most widely used pointing devices in notebook PCs today are the pad, the stick, and the trackball. Each device has its own special characteristics, so it is important that you actually try out and understand a device type, and make sure that it fits your own work and preferences before deciding which to use. The characteristics of each pointing device are described below.

\section{- Pad}

The pad moves the pointer in the direction of your finger motion when you touch it. It is intuitive and an easy device to understand. On the other hand, it can be difficult to tell which part of the finger is touching the pad (corresponding to the pointer), so fine positioning can take some time in getting used to. Some models allow you to do things like sign your name and draw simple diagrams with a pen-like device, which can be convenient depending on the use.

\section{- Stick}

A stick similar to a match extends from the keyboard in between the positions of the "G", "H", and "B" keys as they are located on the "QWERTY" keyboard or the most popular one. The user moves the pointer by exerting pressure on this stick. The pointer moves in the direction of the pressure on the stick, at a speed that varies with the amount of pressure applied. The stick takes longer to get used to than other pointing devices, but can be manipulated comfortably once you are used to it. The most important feature of this pointing device is that you can quickly operate the pointer without moving your fingers from the typing home position. Also, since you use your pointer finger to do the pointing, finepositioning operations may be easy to carry out.

\section{- Trackball}

With this device, the pointer moves in the direction that the ball is rotated, at a speed that corresponds to the speed of the rotation. This device is easy to understand and manipulate, and takes almost no time to get used to. If the ball's diameter is large, then the trackball is a convenient pointing device because moving to distant locations is easy.

\subsection{Working Posture}

"Avoid unnatural postures, and change your posture occasionally"

a. Avoid staying in postures where you are bent too far forward or backward, or twisted, for an extended duration

b. Notebook PC users tend to view the display from too close, so make sure you maintain a distance of at least $40-50 \mathrm{~cm}$ between the display and your eyes (in order to diminish accommodation and convergence effort, the maximum possible distance, according to individual visual capacity and letter/image size, is suggested)

c. Alternate near vision with far vision (i.e. observe object located at least $6 \mathrm{~m}$ far) as frequent as possible

d. Make sure your wrists are not at an unnatural angle

\section{Explanation:}

The ergonomic principle for the posture is that it should be natural and unstrained, so avoid postures where you lean too far forward or backward. Constrained working posture is related to static muscular effort or prolonged state of muscles contractions. The static muscular effort obstructs the blood flow by the internal pressure of the muscle tissue and induces muscle fatigue, which leads to longer resting periods needed than the dynamic muscle effort.

Many users of notebook PCs tend to place them at a diagonal angle to the left or right side if there is not much space on the desktop, and this usually leads to a twisted posture. Do not maintain a posture with only the upper half of the body twisted to one side for extended periods of time. Also, avoid maintaining any single posture for a long time, even if it is a correct, standard posture that places little strain on your body. Make an effort to change your posture from time to time, and stretch your back. Since notebook PCs are small and easy to move, changing the position of your $\mathrm{PC}$ is also an effective method.

Since a notebook PC's display is generally positioned lower than eye level, you will use it in a posture whereby 


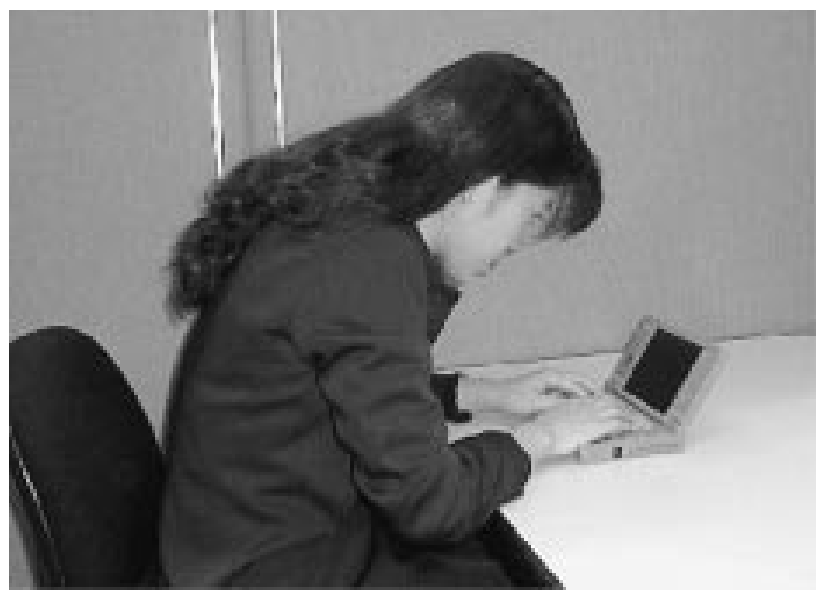

Fig. 12. An example of how not to use a notebook PC (leaning forward too much, with the eyes too close to the display).

you are looking down at the display. Be sure not to bend the neck too much (lean the head too far forward). Also, since the display and keyboard are part of a single unit, you may tend to view the display from too close, so be careful not to let this happen because, as already mentioned, short distance increase substantially (particularly if shorter than $50 \mathrm{~cm}$ ), your accommodation and convergence effort. This, in fact, is believed to be the main source of "occupational asthenopia" in VDT or PC operators. Eye disturbances can be linked, other than to a number of ophthalmic pathologies, also to some minor, but relevant in terms of discomfort, visual and ocular individual problem. The first, frequently due to an overloading of accommodation and convergence, (excess of near vision for that subject in that moment), can be, in most of cases, solved simply watching at far (at least over 6 m) for a few minutes every $1 / 2$ hours. The second, commonly linked to lachrymal film alteration (more frequent in women) and to blinking disorders (mainly incomplete blinking), needs, if suspected, specific ophthalmic checks.

Features of notebook PC keyboards (such as thickness, ability to adjust the angle, and built-in palm rests) vary from model to model. Adjust your posture not only so that it is natural, but also so that it matches the model you are using. In particular, you should avoid using your notebook PC for extended periods of time with an unnatural wrist posture. At the very least, pay special attention to the following two points:

- Is your hand past the wrist bending too far in the upward direction (extension) or the downward direction (flexion)?

- Are your fingertips bent too far to the outside with respect to the wrists (ulnar deviation)?

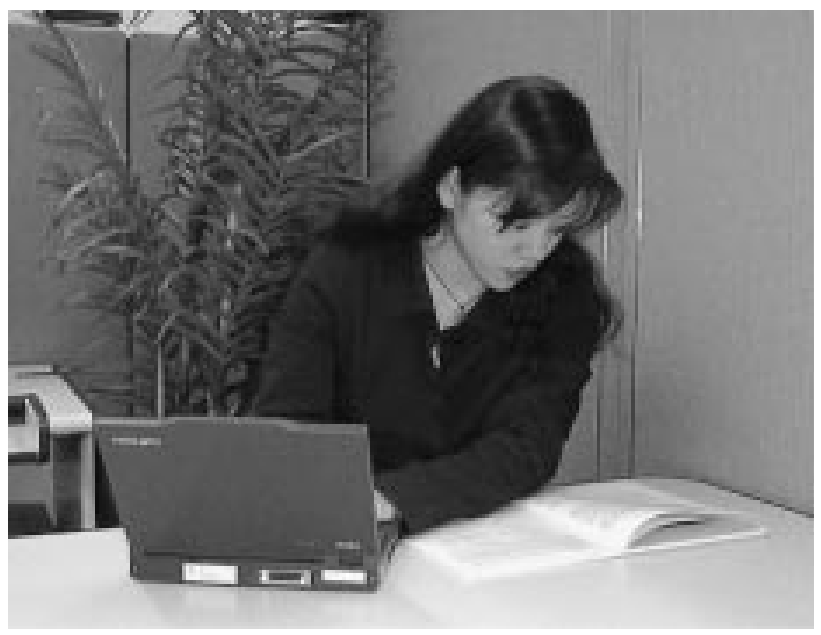

Fig. 13. An example of how not to use a notebook PC (body is twisted).

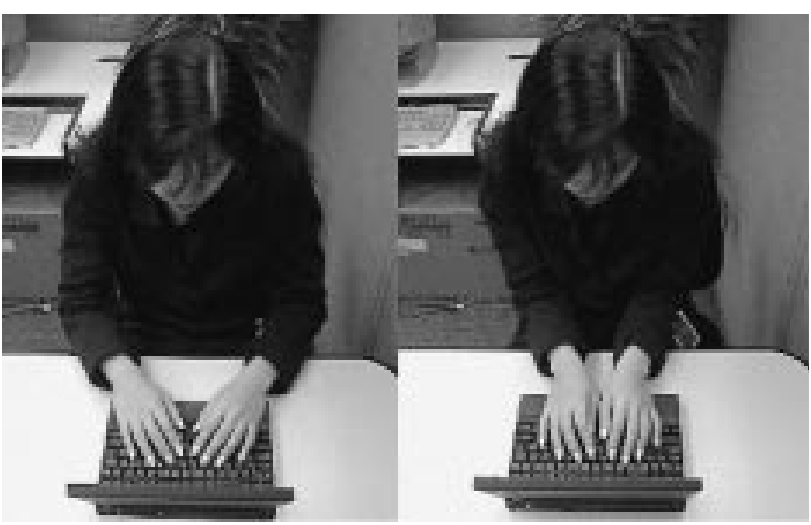

Fig. 14. A posture with the wrists bent in (ulnar deviation) and constricted (right picture), and natural wrist positioning (left picture).

Some notebook PC keyboards have key sizes (key pitches) that are smaller than the key pitch of a standard keyboard $(19 \mathrm{~mm})$. When the key pitch is small, ulnar displacement in particular grows even more severe, and the posture tends to become constricted, so be careful to avoid a strained posture. If you have large hands, for instance, and the keyboard seems small to you, then you will have to alleviate the problem by taking measures such as using an external keyboard, as described later in these guidelines (refer to 4.9).

\subsection{Connecting Peripherals}

"Verify the necessary external peripherals, and set aside space for them"

a. Verify the characteristics of the external peripherals to be connected, such as the sizes and connection locations

b. Connect the peripherals, and allocate space for them to 


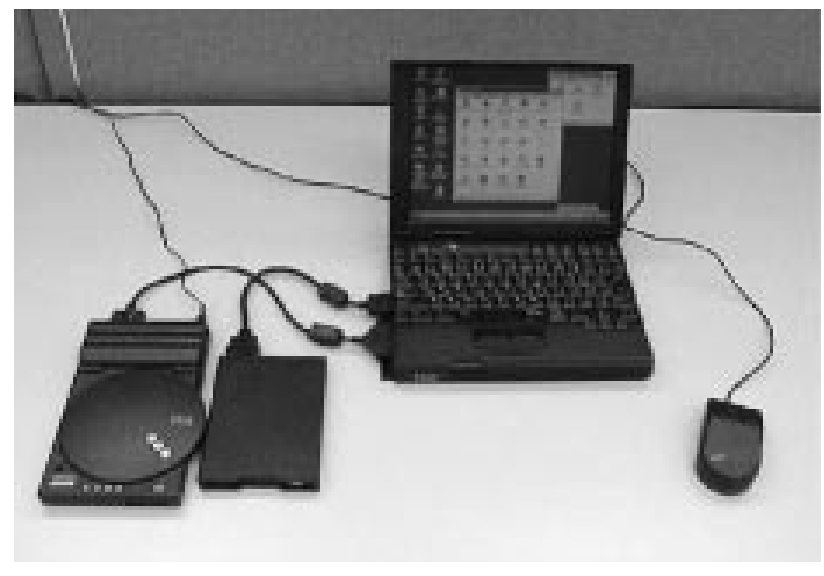

Fig. 15. Peripheral connection example (CD-ROM drive, floppy disk drive, and mouse).

be set up

c. Position each peripheral for ease of use

\section{Explanation:}

Smaller notebook PCs require more peripherals for use on the desktop. Common peripherals include printers, CDROM drives, modems, floppy disk drives, and external numeric keypads. Peripherals can either be connected directly, or via PC cards. Whichever connection method is used, peripherals take up a considerable amount of space, so be sure to set aside space for them in advance. Otherwise, the position of your notebook PC may be restricted, and determined by the peripherals you use. Also, if the printer cable is too short, its length may limit the possible locations for setting up your notebook PC, so make sure that the printer cable is long enough.

After connecting all of the necessary peripherals, verify that they operate correctly. For instance, drives that face to the side or back are extremely difficult to use. Also, verify that the placement of your peripherals does not obstruct the notebook PC's power switch or floppy disk drive, since this may prevent them from operating.

Although one of the advantages of notebook PCs is that they can be easily rotated and moved, but the connection of peripherals can restrict their movement, so you will need to consider the connection of peripherals when setting up your notebook PC. You may want to disconnect all peripherals that you are not using at the time, and only connect them to your notebook PC when you need to use them.

Specialized port replicators and docking stations* are available as add-on options for some models, so you may want to make use of these solutions if necessary.

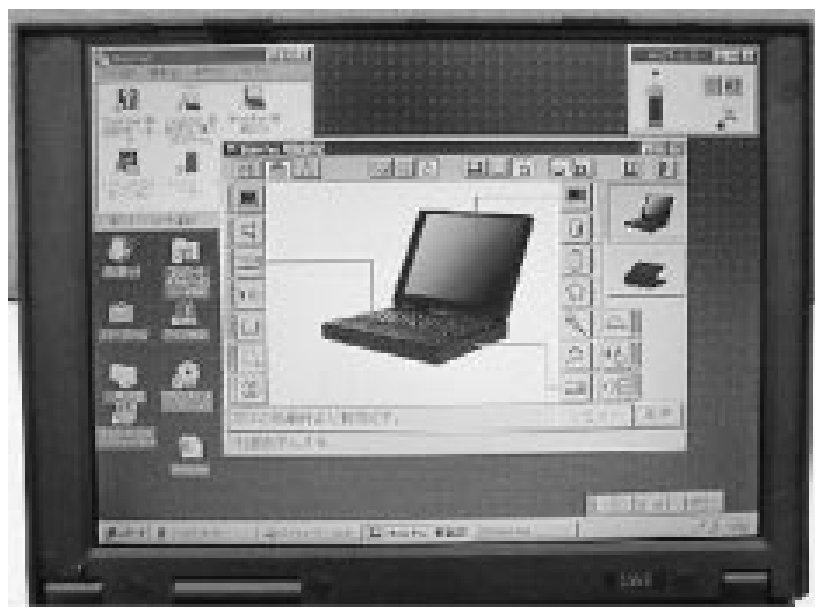

Fig. 16. An example of a program for setting power saving and other functions.

* "Port replicators" and "docking stations" are devices for improving the expandability of a notebook PC by combining with the main unit, in order to allow for the connection of more peripherals, or the use of various expansion boards. However, these devices occasionally make more difficult in operability and usability of the notebook PCs by loss of the advantages such as portability.

\subsection{Function Settings}

"Connect the notebook PC to an electrical outlet, then adjust the energy saving, volume, and other preferences to the optimal settings"

a. Connect the notebook PC to the outlet, and use $\mathrm{AC}$ power as much as possible

b. Understand the power saving functions (power management functions)

c. Adjust the power saving functions, volume, and other preferences to the optimum settings

\section{Explanation:}

Notebook PCs are designed with use of the internal battery in mind, so they come with a variety of power saving functions. In particular, when a notebook PC is run on the internal battery, the display may go blank if there is no input after a certain length of time, and the internal hard disk that contains the user's data may stop spinning temporarily. In most cases, you can set preferences such as the length of time to wait before going into this temporary halt mode (which is called "stand-by mode", "sleep mode", or something similar), and which items to temporarily halt. If 


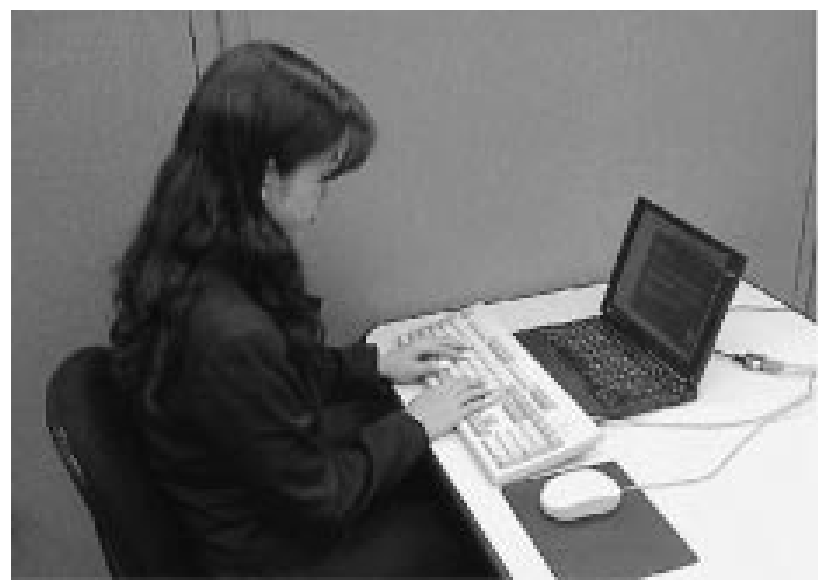

Fig. 17. Example of using a notebook PC with an external keyboard and mouse.

these items are not set correctly, input can slow to a crawl, the display can go blank at inopportune times, and other similar problems can make the notebook PC difficult to use. To use your notebook PC effectively, make sure you understand at least the basics of your notebook PC's power saving functions. Also, some models automatically switch between power saving settings when the notebook PC is run on $\mathrm{AC}$ power or on the internal battery, so if you run it on AC power whenever possible in the office, then you won't have to pay much attention to the power saving functions.

In addition to power saving functions, other settings such as speaker volume and mouse speed can be set according to the user's preferences, so you may want to do so in advance.

\subsection{Using an External Keyboard and an External Display}

"Use correct external keyboards and displays"

a. Verify that an external keyboard and display can be connected

b. If connecting an external keyboard or display improves ease of use, be sure to use the external devices correctly

\section{Explanation:}

Many notebook PCs can be connected to an external keyboard and display. Since notebook PCs are designed with an emphasis on portability, ease of use is occasionally sacrificed. If a small notebook PC is going to be used for a long time, then consideration must be given to ergonomic factors. For instance, the connection of an external keyboard and display can improve ease of use, especially in the following sorts of situations:

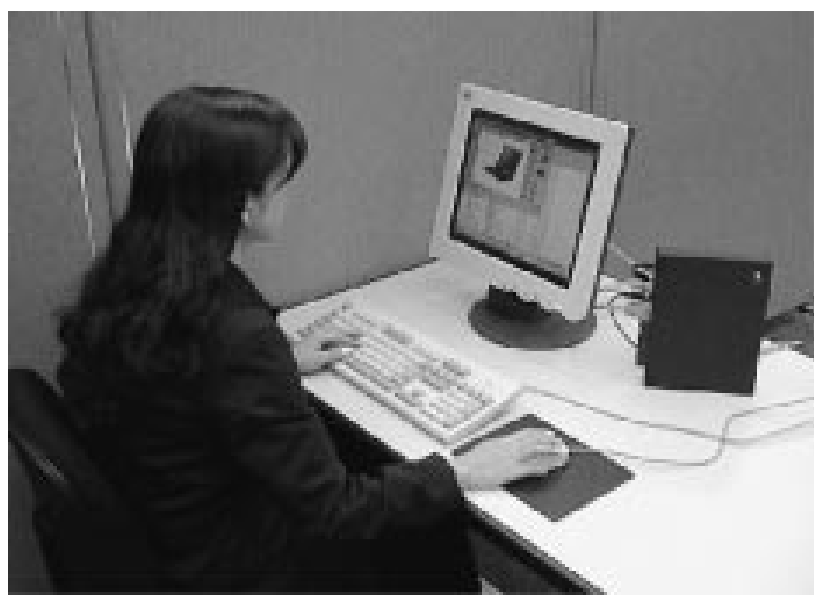

Fig. 18. Example of using a notebook $P C$ with an external keyboard, mouse, and external display connected.

Situations where you might want to attach an external keyboard:

- If the keyboard is difficult to use because the keys are small (less than $1.8 \mathrm{~cm}$ interval from key to key)

- If the keyboard is difficult to use because the home keys are high (3.5 cm or higher off the desktop)

- If the keyboard is difficult to use because the key arrangement is difficult to understand

- If you need to enter many numbers

Situations where you might want to attach an external display:

- If the display is difficult to see because it is small

- If the display is difficult to use because the number of elements or pixels* of the display is small

- If the display is difficult to see because the adjustable brightness or contrast range is insufficient

- When you are viewing animation or video

You may also want to connect only the external keyboard or display, based on your needs. Make sure, however, that if you connect only an external keyboard, that the display is not too far from the eyes as a result. Also, if you connect only an external display, you will have to arrange it so that the notebook PC's built-in display does not get in the way. Furthermore, if the display's settings are incorrect, it may flicker, so check the display and change the settings as necessary.

* "Pixels" refer to the number of pixels that comprise the entire display (such as 640 by 480, or 1024 by 768). The number of pixels displayed on a notebook PC's LCD display is fixed, at 640 by 480,800 by 600 , or another resolution. 


\section{Appendix 1. Characteristics of Notebook PC from the Viewpoint of Ergonomics}

In considering the comfortable use of notebook PCs, it was first necessary to make clear the differences between notebook PCs and traditional desktop PCs. Therefore, while creating these guidelines, we summarized the peculiar ergonomic characteristics of notebook PCs as compared with desktop PCs. One of the original reasons it was decided to create these guidelines was that notebook PCs have many differences when compared to desktop PCs, so the previous guidelines did not always apply. Since knowing the ergonomic issues characteristic of notebook PCs was judged to be a meaningful way of deepening the understanding by users of how to comfortably use notebook PCs, the results of these comparisons are provided below. It is important to understand, however, that not all notebook PCs have the characteristics described below. The characteristics given below were selected because they are absent from typical desktop PCs, and because they are seen in many notebook PCs. Moreover, since PCs are evolving technologically on a daily basis, these characteristics may not completely match the most recent notebook PCs by the time you read this document. Please keep these restrictions in mind as you refer to the characteristics.

It has been noted that when notebook PCs are used in the office, since they emphasize portability, care must be given to a larger number of ergonomic issues than in the case of desktop PCs. Since there are also areas where notebook PCs are actually ergonomically superior, those areas are touched upon here as well.

Appendix 1 (Table) Characteristics of notebook PC from the Viewpoint of Ergonomics

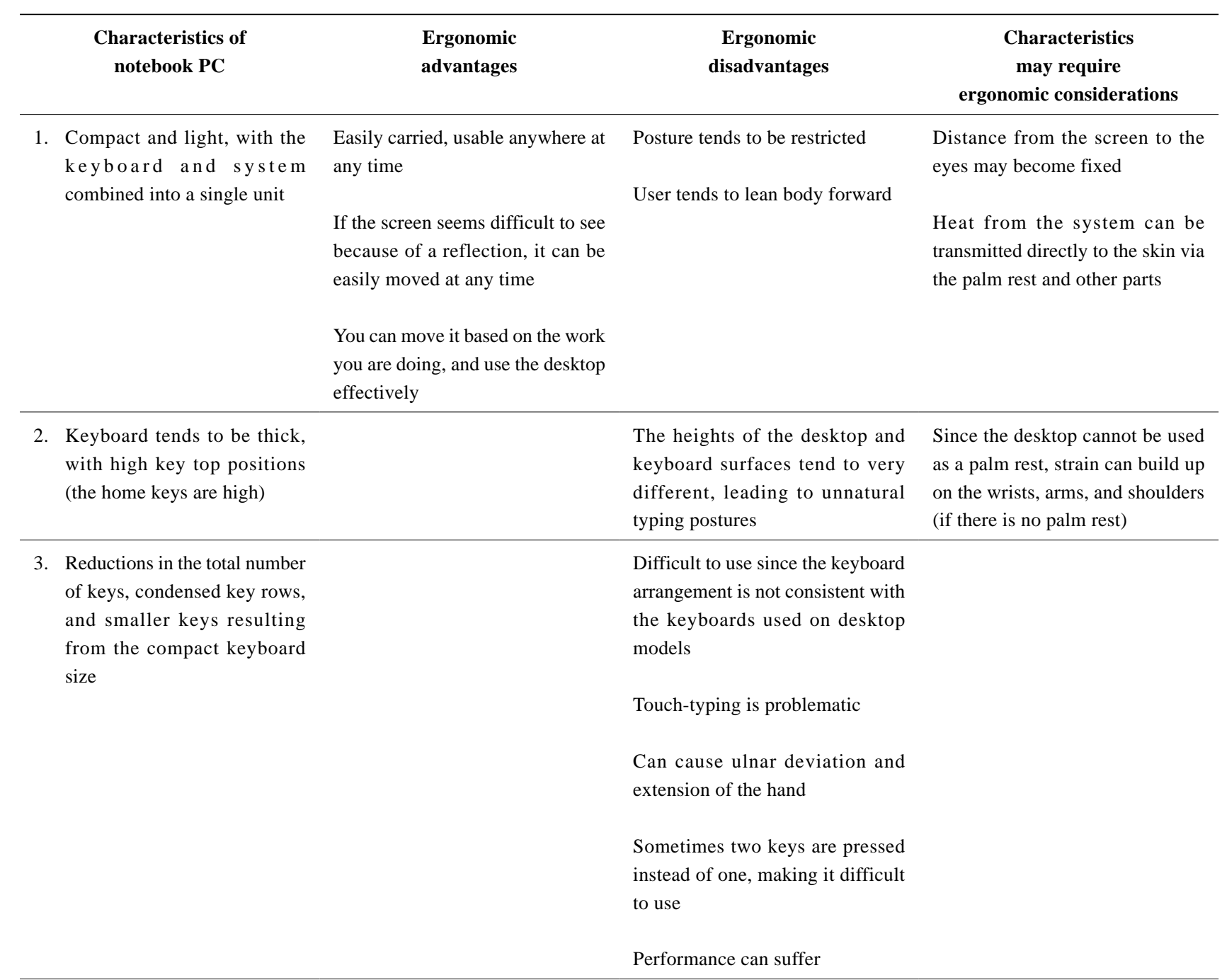


4. Key switch strokes are shallow, and there is less of a "clicking" sensation, but the sound of operation is quieter

5. Keyboard profile (the shape of the keyboard surface) is flat, and key tops are not very concave

6. Affixing a palm rest to the main device
Not much noise caused by

operation
Not much typing feedback, so

performance can suffer
Two keys are often pressed simultaneously
Since the required depth is available, by adjusting the chair's height as appropriate, the notebook PC can be set up so that the wrists can be rested

When using the notebook PC on the notebook, it is easy to use with the palm rest supporting the main device

7. Pointing device is not a mouse

Easy to use while typing (since maintaining the fingers in the home key position is easy)

Display surface is flat and has no distortions whatsoever

No blur or smearing, so the image is sharp

8. Display surface is flat and has no distortions, so there is no blur or smearing (LCD)
If the keyboard is thick and the chair is low, the palm rest may get in the way
Since the space available for pointing is small, operating the pointing device may be difficult
User posture may be unnatural during dragging
May not be possible to raise the brightness/contrast to the optimum level when used in a bright location

May not be possible to lower the brightness/contrast to the optimum level when used in a dark location

\section{Display's reflection ratio is low \\ Contrast and sharpness do not decrease as much as on a CRT in a bright place}

11. Display's position is low, and often tilted upward

Line-of-sight angles downward, so the eyes do not dry out very much
Field of vision is wide, so there is not much sense of confinement

\footnotetext{
12. Fast-moving images blur (only in the case of certain STN type LCDs)
}

\section{Ceiling lights tend to reflect on the} screen
Position of the mouse cursor can

be lost, reducing performance

Blurring stands out when

animations are displayed, making

them difficult to view

\footnotetext{
13. Linearity of the display's gray scale is poor
}

Distinguishing between the gray scales in the high luminance level can be impossible 


\begin{tabular}{lll}
\hline $\begin{array}{l}\text { 14. Display looks different } \\
\text { depending on the angle of view } \\
\text { (range of viewing angles is } \\
\text { narrow) }\end{array}$ & $\begin{array}{l}\text { It is difficult for onlookers to see } \\
\text { what you are working on }\end{array}$ & $\begin{array}{l}\text { Uniformity of brightness or color } \\
\text { may look poor }\end{array}$ \\
\hline $\begin{array}{l}\text { 15. Power consumption is } \\
\text { minimal, and detailed power } \\
\text { management preferences can }\end{array}$ & so it is easy on the environment \\
be set & Relatively quiet & Shades may invert \\
& & $\begin{array}{l}\text { Screen sometimes darkens during } \\
\text { battery use }\end{array}$ \\
& & $\begin{array}{l}\text { Depending on the settings, the hard } \\
\text { disk may spin down too often, } \\
\text { making it difficult to use } \\
\text { (especially during operations such } \\
\text { as text input) }\end{array}$
\end{tabular}

\section{Appendix 2. Committee members on Flat Panel Display Ergonomics in Japan Ergonomics Society (JES)}

Satoru Abe

Masahiro Akutsu

Toyohiko Hatada

Yoshinori Horie

Yoshihiko Nakano

Shin Saito

Susumu Saito (Chair)

Midori Sotoyama

Ryozo Yamashita

Ryoji Yoshitake
Appendix 3. Technical committee members on Human-Computer Interaction, International Ergonomics Association (IEA)

\author{
Ulf Bergqvist (Sweden) \\ Tadahiko Fukuda (Japan) \\ Yoshio Hayashi (Japan) \\ Nicolas V. Marmaras (Greece) \\ Alexander Nikov (Bulgaria) \\ Bruno Piccoli (Italy) \\ Susumu Saito (Chair: Japan) \\ Michael J. Smith (U.S.A.) \\ Midori Sotoyama (Japan) \\ Tom Stewart (U.K.) \\ Glenn Sweitzer (U.S.A.) \\ Sasitorn Taptagaporn (Thailand) \\ Maria Beatriz G. Villanueva (Philippines) \\ Ryozo Yamashita (Japan) \\ Ryoji Yoshitake (Japan)
}

